\title{
Assessment of Patients' Medical Laboratory Request Forms for Compliance in Jos University Teaching Hospital, Jos-Nigeria
}

\author{
Olaniru B Olumide ${ }^{1}$, Alla 0 Joel $^{2}$, Michael E Paul ${ }^{1}$, Nedolisa Angela C ${ }^{3}$, Oyero Steve $\mathrm{K}^{4}$, Obeta $\mathrm{M}$ \\ Uchejeso $^{5 *}$, Isiguzoro Ikechukwu OU ${ }^{1}$, Iheanacho U Charity ${ }^{2}$, Ntuhun B Dennis ${ }^{2}$, Fiyaktu Bwotle \\ Yakubu1', Jugu P Kizito4, Ogunbiyi A Emmanuel ${ }^{3}$ and Song Elisha² \\ ${ }^{1}$ Department of Chemical Pathology, Jos University Teaching Hospital, Nigeria
}

${ }^{2}$ Department of Haematology and Blood Group Serology, Jos University Teaching Hospital, Nigeria

${ }^{3}$ Department of Medical Microbiology, Jos University Teaching Hospital, Nigeria

${ }^{4}$ Department of Histopathology, Jos University Teaching Hospital, Nigeria

${ }^{5}$ Department of Chemical Pathology, Federal School of Medical Laboratory Science, Jos, Nigeria

*Corresponding author: Obeta M Uchejeso, Department of Chemical Pathology, Federal School of Medical Laboratory Science, Jos, Nigeria.

To Cite This Article: Obeta M Uchejeso, Assessment of Patients' Medical Laboratory Request Forms for Incomplete Data in Jos University Teaching Hospital, Jos-Nigeria. Am J Biomed Sci \& Res. 2019 - 6(4). AJBSR.MS.ID.001056. DOI: 10.34297/AJBSR.2019.06.001056.

Received: 監 November 19, 2019; Published: 掣 December 04, 2019

\begin{abstract}
Background: Medical laboratory request forms are important medium of communication between the physicians and the medical laboratory practitioners. The request form provides demographic information of the patients as well as clinical conditions, clinician, specimen information and the targeted laboratory. Inability of the physician to indicate any of the information mentioned above could pose a negative effect in accurate definitive diagnosis of the patients' disease which could affect proper treatment or management.
\end{abstract}

Aim of study: The study seeks to assess the level of completeness of patients request forms in Jos University Teaching Hospital.

Study volunteers and methods: A retrospective study was carried out on 4,310 in and out Patients request forms as filled by the physician and sent to four laboratory departments of the hospital between the period of January and July 2017 having gotten Ethical clearance from the ethical committee of the teaching hospital.

Results: The result of the findings revealed that from the 4310 request forms assessed, patient' demographic information, recorded $20.9 \%$, $17.2 \%, 14.9 \%, 1.3 \%, 0.9 \%$ and $0.0 \%$ incomplete rate for age, ward, department, hospital number, sex and name of the patient respectively. Patients' clinical information revealed $54.7 \%$ and $8.0 \%$ incomplete rate for patients' clinical data and provisional diagnosis respectively. For Clinician information, incomplete rate of $54.7 \%, 17.8 \%$ and $8.2 \%$ was recorded for signature of the doctor, name of doctor and the consultant respectively. On specimen information, an incomplete rate of $98.2 \%, 9.0 \%, 7.6 \%$ and $2.9 \%$ was observed for time of specimen collection, date, nature of specimen and investigation required respectively. On targeted laboratory, the study recorded an incomplete rate of $8.6 \%$. The result also show that from all the 4310 request form assessed, none is $100 \%$ completely filled.

Conclusion: This study reveals incomplete filling of medical laboratory request forms in JUTH. There is the need to adhere to International Standard Organization guidelines in filling the laboratory request forms for a better patient care and improvement of quality since most decision are taken by the physician or the nurse depends on medical laboratory results.

Keywords: Assessment; Data; Medical Laboratory; Request Forms

\section{Introduction}

Medical Laboratory testing plays a very important role in the detection, diagnosis and treatment of a patient's disease. The timely

result generated in the medical laboratory guides the physician or the nurse in decision making pertaining to the treatment, 
discharge and/ or the management options for a patient $[1,2]$. An estimated $60-70 \%$ of all decisions regarding a patients' diagnosis and treatment, hospital admission and discharge are based on the medical laboratory results [3]. In a hospital setting, patients' medical laboratory tests are conducted based on request made on a medical laboratory request form filled by the Physician or the Nurse in some cases [4]. The medical laboratory request form in the Jos University Teaching Hospital's contains demographic information such as patients' full name, Age, Sex, Hospital Number, Specimen,
Tribe, Ward, Department, Clinical Data, Provisional Diagnosis, Investigation Required, Date, and Time of sample Collection, Consultant's Name, Name and Signature of the Doctor. Research has it that most medical laboratory errors emanate from the preanalytical phase of diagnosis [5]. Among these, is filling of medical laboratory request forms, where adequate information provided plays a vital role in the definitive diagnosis thereby encouraging quality management system and international standard (Figure 1).

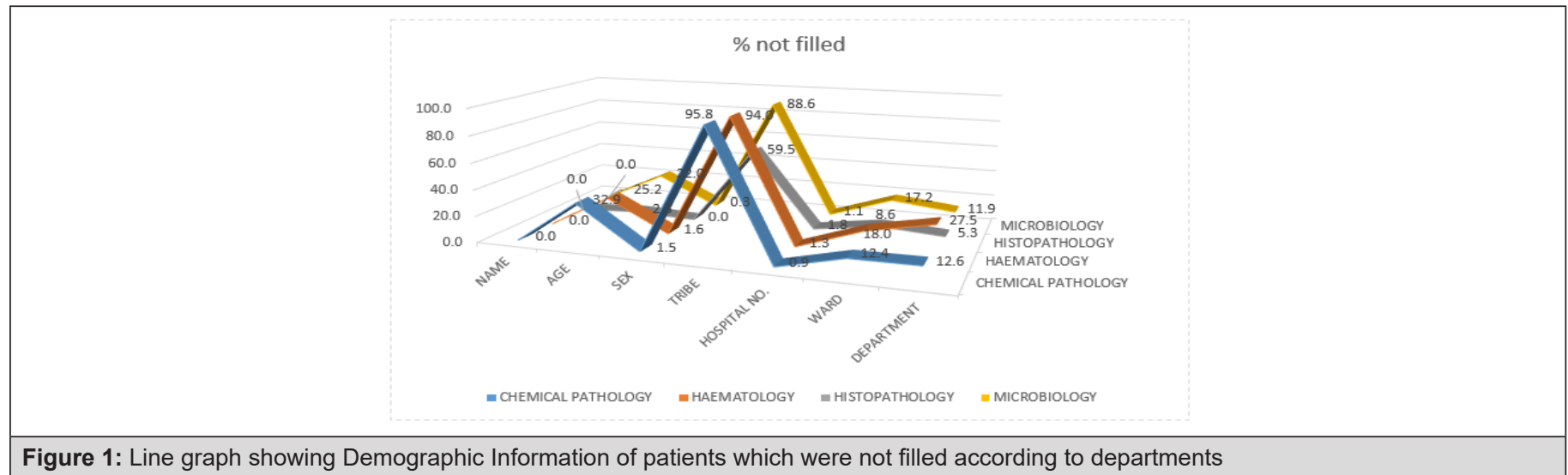

The inability of the Doctor to indicate any of the information above could pose a negative effect in accurate definitive diagnosis of the patient's disease and could affect proper treatment or management [6].

This study therefore seeks to assess the level of completeness of patients' medical laboratory request forms in Jos University Teaching Hospital, since there has been no study on the trend of incompletely filled patients' medical laboratory request forms in the tertiary hospital.

\section{Materials and Methodology}

This research was carried out on 4,310 in and out patient request forms which had been filled by the physicians and sent to any of the four components of medical laboratory department of Jos University Teaching Hospital (JUTH), a 500 bed tertiary hospital in Jos, the Plateau state capital in north central Nigeria. JUTH provides a measure of emergency, inpatient and outpatient services. These include medical, surgical, laboratory, emergency/ trauma, obstetrical, gynaecological, and paediatric services. This retrospective study was carried out on request forms received in the medical laboratories between the period of January and July 2017. Sixteen variables including patients' full Name, Age, Sex, Hospital Number, Tribe, Ward, Department, Clinical Data, Provisional Diagnosis, specimen, Investigation Required, Date, Time of sample Collection, Consultant's Name, Name and Signature of the Doctor were assessed in all the randomly retrieved request forms in each of the laboratory and were analysed using SPSS version 21 computer programme. Ethical approval was sought and gotten from the ethical committee of Jos University Teaching Hospital.

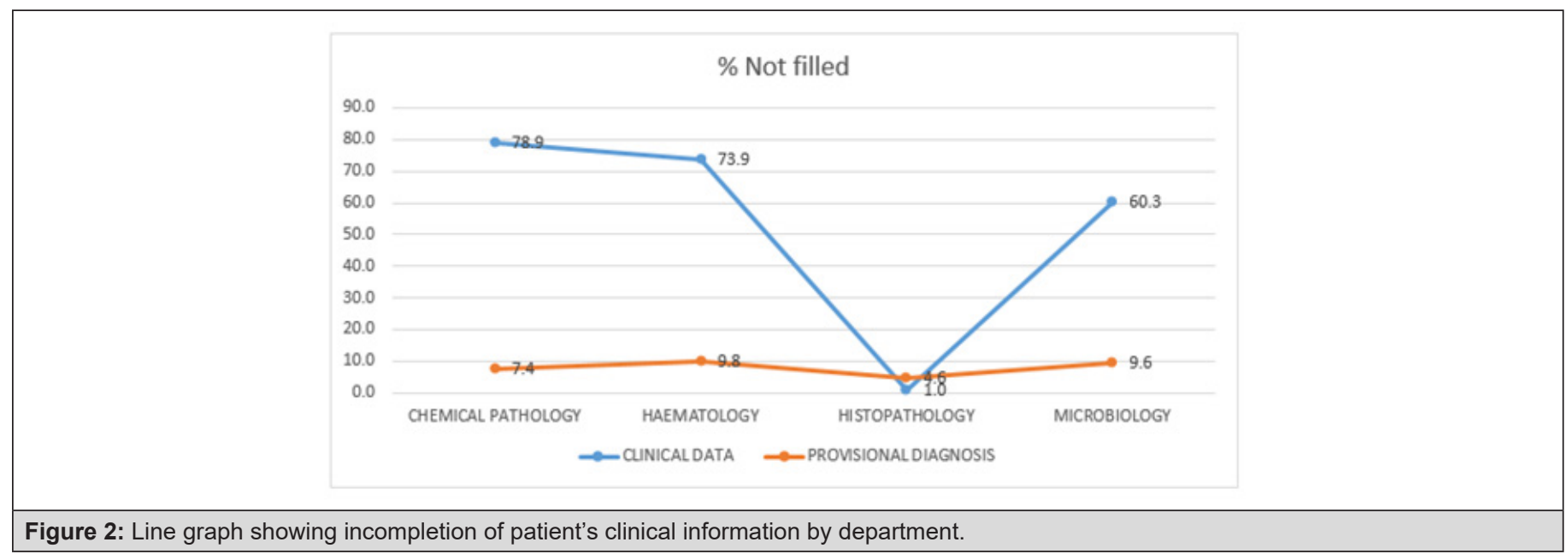




\section{Result}

The table below revealed that following the study of the extent of incompletion of data in patients' laboratory request forms, demographic information in the request form which comprises of name of the patient had an $100 \%$ completion in all the four (4) laboratories. Data acquired from age revealed that the incompletion rate in Chemical pathology was 32.9\%, Haematology was $25 \%$, Histopathology was $2.3 \%$, and Microbiology was $22 \%$. In regard to gender, Chemical pathology had an incompletion rate of $1.5 \%$, Haematology had 2\%, and Histopathology had $0 \%$ while Microbiology had $0.3 \%$ Figure 1. With respect to Tribe, Chemical pathology laboratory had an incomplete rate of 96\%, Haematology had 94\%, Microbiology had 89\% and Histopathology had 59.5\%. Result on the incompletion rate of Hospital number on the request form shown that Chemical pathology laboratory had an incomplete rate of $0.9 \%$, Microbiology had 1\%, Haematology with $1 \%$ and Histopathology with $1.8 \%$. Incomplete rate on Ward of the patient revealed that Chemical pathology laboratory had $12 \%$, Haematology had 18\%, Histopathology 8.6\% and Microbiology had $17 \%$. As for the Department of the patient, the incomplete rate in Chemical pathology laboratory was $13 \%$, Haematology was $28 \%$, Histopathology was $5.2 \%$ and Microbiology was $12 \%$ (Table 1).

\begin{tabular}{|c|c|c|c|c|}
\hline \multirow[t]{2}{*}{ Parameter } & \multicolumn{4}{|c|}{ Departments } \\
\hline & Med. Micro & Histopathology & Haematology & Chemical pathology \\
\hline Demographic information & $\%$ unfilled & $\%$ unfilled & $\%$ unfilled & $\%$ unfilled \\
\hline Name & 0 & 0 & 0 & 0 \\
\hline Age & 22 & 2.3 & 25 & 32.9 \\
\hline Sex & 0.3 & 0 & 2 & 1.5 \\
\hline Hospital Number & 1 & 1.8 & 1 & 0.9 \\
\hline Tribe & 89 & 59.5 & 94 & 96 \\
\hline Ward & 17 & 8.6 & 18 & 12 \\
\hline Department & 12 & 5.3 & 28 & 13 \\
\hline \multicolumn{5}{|c|}{ Clinical Information } \\
\hline Clinical Data & 60 & 1 & 74 & 79 \\
\hline Provisional Diagnosis & 10 & 4.6 & 10 & 7 \\
\hline \multicolumn{5}{|c|}{ Specimen Information } \\
\hline Nature of Specimen & 8 & 2 & 11 & 8 \\
\hline Investigation Required & 2 & 6.6 & 2 & 0.7 \\
\hline Date of collection & 5 & 9 & 6 & 13 \\
\hline Time of Specimen Collection & 100 & 95.3 & 99 & 99.3 \\
\hline \multicolumn{5}{|c|}{ Clinician Information } \\
\hline Consultant's Name & 9 & 2.8 & 12 & 8 \\
\hline Name of Doctor & 15 & 31 & 15 & 11 \\
\hline Doctor's Signature & 43 & 73 & 48 & 55 \\
\hline \multicolumn{5}{|c|}{ Lab Information } \\
\hline Targeted Laboratory & 9 & 0 & 15 & 9 \\
\hline
\end{tabular}

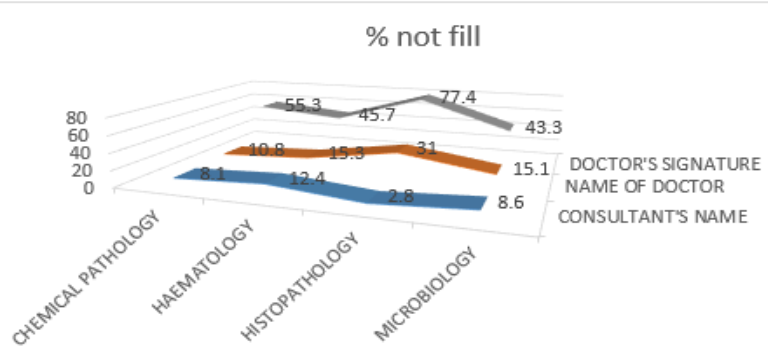

$\because$ CONSULTANT'S NAME $\quad$ NAME OF DOCTOR $\because$ DOCTOR'S SIGNATURE

Figure 3: Line graph showing Level of Incompletion of Patients' Clinician information in the request forms. 
For the result on clinical information, clinical data shows an incomplete rate of $60 \%, 1 \%, 74 \%$, and 79\% in Medical Microbiology, Histopathology, Haematology and Chemical Pathology Laboratory respectively. Provisional Diagnosis showed an incomplete rate of $10 \%, 4.6 \%, 10$ and $7 \%$ for Microbiology, Histopathology, Haematology and Chemical Pathology Laboratory respectively Figure 2. The Table also shows the incompletion rate of patients clinician information in the request form. On Consultant's name, the result revealed an incomplete rate of $9 \%$ in Microbiology Laboratory, 2.8\% in Histopathology Laboratory, $12 \%$ in Haematology laboratory and $8 \%$ in Chemical pathology Laboratory. On the Incompletion rate of the name of doctor, the table shows an incomplete rate of $15 \%, 31 \%, 15 \%$ and $11 \%$ for Microbiology, Histopathology, Haematology and Chemical Pathology Laboratory respectively. Signature of the Doctor recorded 43\% in Microbiology Laboratory, 73\% in Histopathology, 48\% in Haematology laboratory and 55\% in Chemical Pathology Laboratory. On specimen information, the result on the table shows an incomplete rate of $8 \%$ for the nature of specimen in Microbiology laboratory, 2\% in histopathology, 11\% in Haematology laboratory, and 8\% in Chemical Pathology Laboratory (Figure 3).

'Investigation required' shows and incomplete rate of $2 \%$, 6.6\%, 2\% and $0.7 \%$ in Microbiology, Histopathology, Haematology and Chemical Pathology Laboratory respectively. Date of Specimen collection recorded and incomplete rate of 5\% in Microbiology Laboratory, 9\% in Histopathology, 6\% in Haematology and 13\% in Chemical Pathology Laboratory. Time of specimen collection revealed an incomplete rate of 100\%, 95.3\%, 99 and $99.3 \%$ in Medical Microbiology, Histopathology, Haematology and Chemical Pathology Laboratory respectively. On the 'Targeted Laboratory Information, It was observed that Chemical pathology laboratory had an incomplete rate of 9\%, Haematology had 15\%, Histopathology had $0 \%$ and Microbiology laboratory had 9\%.

\section{Discussion}

Accurate Laboratory diagnosis begins with proper and adequate filling of patient's laboratory request form by the physician or the nurse. It is in line with this reality that the International Organization for Standardization [7] for Medical Laboratories states that the laboratory test request form should contain sufficient information to identify the patient, the authorized requester as well as significant clinical information. This study was carried out to assess the level of incomplete data on the laboratory request forms sent to Haematology, Chemical Pathology, Medical Microbiology and Histopathology Department of the Jos University Teaching hospital, Plateau, Nigeria. The data revealed that of all the 4310 request form distributed, none is $100 \%$ completely filled; either one parameter is missing or the other. The study also reveals that of all the 4,310 laboratory forms assessed; only the name of the patients was completely filled in all the forms.
Our findings appear to be in accordance with similar studies conducted in other settings by Adegoke \& Idowu, [8], Olayemi \& Asiamah-Broni [6], Burton \& Stephenson [9], in which only the name of the patients was completely filled, this is not startling because of the importance affix to a person's name in our society and it is also very likely that the sample would not be accepted by the laboratories if the patients name was absent. Hence, the requesters were subservient in filling the name in all the request forms. Oladiende and colleagues [10] observed that name of the patient and the name of the attending physician were completely filled in all the forms they analysed. Paingha \& Ezenwa [11] revealed that the name of the patient and the investigation requested for were filled out in all the request form they evaluated.

\section{Age}

We found out that $20.9 \%$ of the request forms evaluated did not have the age of the patient, this is a big challenge for proper research and epidemiological studies, apart from the fact that certain parameters have difference age ranges. This may be due to some clinicians lack of recognition of age as a prerequisite to accurate diagnosis Most often, many a clinician sees the age demography as an information to overlook in cases of emergency when samples are to be sent to the laboratory.

\section{Tribe}

$85.1 \%$ of the request forms assessed did not have the tribe of the patients, and this is a serious problem to epidemiological studies and can hinder prompt intervention against diseases which are common among people from certain tribal divide.

\section{Ward and Department}

$17.2 \%$ and $14.9 \%$ of the request forms did not have the ward and the department of the patients respectively. This limitation is effectual in deterring the onward transmission of results of severe illnesses with critical values been that the laboratory users will spend time in searching for the patient's location within the hospital. Incompletely filled patient laboratory request forms contribute immensely to pre-analytical errors thereby hindering accurate analytical and post analytical procedures. Patients' demographic and clinical information are of essence, it aids in specimen identification and proper interpretation of result. In situation where samples from different patients have the same or similar names, other demographic such as tribe, hospital number, age, sex, are crucial in identifying and in sorting out the patient and the sample. Also reference ranges of certain parameters differs with age, sex and even location and tribe.

\section{Clinical Data}

$54.7 \%$ of the request forms assessed were without clinical data. This is far higher than that of Oladiende and co-workers [10] work with an incomplete error of $6.7 \%$ and that of the study conducted by Karunanandham et al. [12] which recorded an incomplete error 
of $46.7 \%$ and Nutt et al. [13] with an incomplete rate of $19.1 \%$. This is however a little bit lower than that of the studies of Kapil et al. [14] with non- conformity of 57.80\%. Inclusion of patient's clinical data in the laboratory request forms helps the laboratory users in several ways than one. It helps the laboratory users in putting the result of laboratory findings in the right perspective and to question laboratory interferences when unusual results are gotten. It also aids the Medical Laboratory Scientists in the interpretation of the outcome of investigations which helps in giving quick attention to dispatching of abnormally high or low value results. A quality and well filled clinical data also, reduces the repetition of test and cost.

\section{Time of Specimen Collection}

$98.2 \%$ of the assessed request forms did not have the time of specimen collection. This is a frightening figure owing to the importance this plays in laboratory diagnosis of certain parameters. The time of sample collection is vital in calculating the turnaround time (TAT) of test. Results of some laboratory investigations are time dependent as seen in glucose estimation. Time of specimen collection is also needed to resolve issues of ambiguous results which could be due to a prolonged time between the time of sample collection and the time of sample separation or analysis as often seen in arterial blood gas analysis, electrolytes estimation and pus cells in urine microscopy.

\section{Conclusion}

In the assessment of incompleteness of medical laboratory forms in a tertiary hospital in Jos, the analysed data revealed that of all the 4310 request forms distributed, name of patients was $100 \%$ filled in the medical laboratory request forms assessed, while the parameter that was least filled is Time of Specimen Collection followed by Tribe. Chemical pathology and Haematology request forms recorded high incompleteness while request forms sent to Histopathology laboratory recorded High Completeness because they have better practicable guidelines for sample rejection on the basis of incompleteness of request forms. The prominent difficulty offered by incomplete request forms is the capacity to handicap proper definitive diagnosis in short-turn-around time thereby reducing the quality management system component of health practice score line in Jos University Teaching Hospital.

From this study, it is hereby recommended that:

a) There is the need to develop a laboratory quality and practicable guidelines for sample rejection on the basis of incompleteness of request forms.

b) There should be proper orientation/training by medical laboratory scientist to all new house officers and resident doctors on the imperative of proper filling of laboratory request forms.

c) There is a need to have an annual joint physician - medical laboratory staff conferences to deliberate on issues relating to the duo in improving the standard and quality of laboratory results to enhance quality healthcare delivery to the patients.

d) Consultants should ensure that house officers and resident doctors under their supervision develop the habit of filling the request forms adequately.

e) Regular assessment of medical laboratory request forms should be carried out to improve the health care delivery [15].

f) Provision for patients and doctors phone number should be included in the request forms as this will enable the medical laboratory users to place a call on either the patient or the doctor's as the case may require.

\section{Acknowledgement}

The following Medical Laboratory Scientists are highly acknowledged for assistance during the data gathering and sorting of medical laboratory request forms from their various departments: Antum Sanda, Emem Tom, Sarah Mwanse, Caroline Andrew, Zingdam Nanfa, Hangdap Samuel, Sati Tongyriang, Thamos Whi, Lois Daniel, Jonathan Dikop, Kwanmen Tracy and Dajan Wilfred.

\section{References}

1. Nitin Kaushik, Sol Green (2014) Pre-analytical errors: their impact and how to minimize them. Medical Laboratory Observer p. 3-5.

2. Obeta MU, Maduka KM, Ofor IB, Ofojekwu NM. (2019) "Improving Quality and Cost Diminution in Modern Healthcare Delivery: The Role of the Medical Laboratory Scientists in Nigeria". International Journal of Business and Management Invention (IJBMI) 8(3): 8-19.

3. Mayo Clinic School of Health Science (2016) Medical Laboratory Sciences.

4. Hammerling J (2012) A review of medical errors in laboratory diagnostics and where we are today. Lab Med 43(2): 41-44.

5. Lippi G, Becan-McBride K, Behulova D, Bowen R A, Church S, et al. (2013) Preanalytical quality improvement: In quality we trust. Clin Chem Lab Med 51(1): 229-241.

6. Olayemi E, Asiamah-Broni R (2011) Evaluation of request forms submitted to the haematology laboratory in a Ghanian Tertiary hospital. Pan African Medical Journal 33: 233-240.

7. International Organization for Standardization. ISO 15189: Medical Laboratories-Particular requirement for Quality and Competence. Geneva: International Organization for Standardization.

8. Adegoke OA, Idowu AA, Jeje OA (2011) Incomplete laboratory request forms as a contributory factor to pre-analytical errors in a Nigerian teaching hospital. Afr J Biochem Res 14: 82-85.

9. Burton JL, Stephenson TJ (2001) Are Clinician failing to supply adequate information when requesting a histopathological investigation? Journal of Clinical Pathology 54(10): 806.

10. Oladiende BH, Omeregie R, Osakue EO, Onifade AO (2012) Evaluation of laboratory request forms for incomplete data at rural tertiary hospital in Nigeria. NZJ Med Lab 66(2): 39-41.

11. Paingha Joe Alagoa, Ezenwa Patrick Udoye (2015) Laboratory request forms - how well do Doctors fill them? A look at the Practice at the Niger Delta University Teaching Hospital, Okolobiri, Bayelsa State, Nigeria. The Nigerian Health Journal. 
12. Karunanandham S, Rajappa T, Jesudoos S, N Naveethalakshmi (2015) A study of adequacy of completion of clinical biochemistry laboratory request forms 3: 1378-1382.

13. Nutt L, Zemlin AE, Erasmus RT (2008) Incomplete laboratory request forms: The extent and impact on critical results at a tertiary hospital in South Africa. Ann Clin Biochem 45: 463-466.
14. Kapil Bhatia, Palavi Bhatia, Manasvi Praveen Kumar (2017) Assessment of Biochemistry Laboratory requisition forms as a contributory factor to preanalytical errors in a tertiary care teaching hospital. International Journal of Contemporary Medical Research 1(5): 77-83.

15. Burnett L, Chesher D, Mudaliar (2004) Improving the quality of information on pathology request forms. Ann Clin Biochem 41: 53-56. 